\title{
On the Interaction of Bovine Serum Albumin (BSA) with Cethyltrimethyl Ammonium Chloride Surfactant: Electron Paramagnetic Resonance (EPR) Study
}

\author{
Marcel Tabak ${ }^{1}$, Diógenes de Sousa Neto ${ }^{1,2}$, and Carlos Ernesto Garrido Salmon ${ }^{1,2}$ \\ ${ }^{1}$ Instituto de Química de São Carlos, Universidade de São Paulo, \\ Cx. Postal 780, CEP 13560-970, São Carlos, SP, Brazil and \\ 2 Instituto de Física de São Carlos, Universidade de São Paulo, \\ Cx. Postal 66318, CEP 05315-970, São Carlos, SP, Brazil
}

Received on 1st August, 2005; accepted on 31 October, 2005

\begin{abstract}
Electron paramagnetic resonance (EPR) has been used to monitor the interaction of bovine serum albumin (BSA) with cationic cethyltrimethylammonium chloride (CTAC) at pH 7.0. EPR results using 5-DSA and 16-DSA nitroxide spin labels show that in the presence of BSA the EPR spectra are composed of two label populations, one contacting the protein and a second one due to label localization in the micelles. Evidence is also obtained for a competition of the surfactants with the spin labels for the high affinity binding sites of the stearic acid spin labels as monitored by changes in the fraction of the two label populations as the surfactant concentration is increased. The effect of sodium dodecylsulfate (SDS) reported previously seems to be stronger in the sense that increase in SDS concentration leads to a complete transfer of spin label from close protein contact sites to the micelles while for CTAC, apparently, a significant immobilization of probe remains even at higher surfactant concentrations. EPR gives information on the dynamics inside the protein-surfactant aggregates and associated to label localization and motion. The dynamics of the nitroxide spin-labels bound to the protein correlate to the stronger binding of SDS to BSA as compared to CTAC binding. Simulation of EPR spectra for spin labels in pure CTAC micelles, in pure protein or in protein-bound micelles show rotational correlation times similar to those obtained from the simple evaluation based on the intensities of nitrogen hyperfine coupling components. Rotational correlation times obtained for 5-DSA bound to protein are larger as compared to 16-DSA values suggesting greater mobility for the later even when bound to the protein.
\end{abstract}

Keywords: EPR; Bovine serum

\section{INTRODUCTION}

Serum albumin consists of a globular protein synthesized by the liver in mammals, corresponding to the most abundant protein in serum, accounting for $60 \%$ of the total globular protein in blood plasma [1-4]. It is associated to the binding and transport of several small molecules such as fatty acids, dyes, metals, amino acids, drugs, as well as several pharmaceutical compounds [1-5]. Bovine serum albumin (BSA) is a very interesting biophysical and biochemical system and has been quite studied over the last 40 years. Its primary structure is very well known consisting of 585 amino acid residues, whereas its secondary structure contains $67 \%$ of alpha helix and 17 disulfide bridges that confer to the protein a remarkable stability $[1,3]$.

The interaction of globular proteins, especially BSA, with surfactants, in particular sodium dodecyl sulfate, SDS, has been extensively studied aiming to understand how surfactant binding affects the protein structure and function [6]. Two interesting ranges of surfactant concentrations associated to different effects on the interaction with the protein are worthy of mention: the first one, at stoichiometric surfactant concentration, is related to the binding that occurs to specific high energy sites on the protein, being the interactions, in general, of electrostatic nature; the second one is related to higher surfactant concentrations, above the critical micellar concentration $(\mathrm{cmc})$, where a massive increase in binding due to cooperative ligand interactions takes place [7-9]. The protein denaturation is believed to occur at these concentrations. Several techniques have been used to investigate the assembled protein-surfactant complexes: UV-Vis absorption [10], fluorescence and circular dichroism [7,10-13], small angle neutron scattering (SANS) [14,15], small angle X-ray scattering (SAXS) $[16,17,18]$, light scattering $[19,20]$, cryogenic-transmission electron microscopy and ${ }^{2} \mathrm{H}$ NMR relaxation rates [21,22] and other physicochemical techniques such as tensiometric and viscosimetric measurements [23], isothermal titration calorimetry and FTIR [24] and differential scanning calorimetry [25]. At high surfactant concentrations a variety of models have been proposed, being the "pearl necklace" model the most discussed in literature [7-9]. In such a model, the polypeptide chain forms the string of the necklace and micelle-like aggregates are randomly wrapped along the unfolded protein polypeptide chain [7-10,14,15].

In recent years the interaction of serum albumin with ionic surfactants has been studied by our group. In this context, a spectroscopic study involving the interaction of both BSA and HSA with the anionic sodium dodecyl sulfate (SDS), the zwitterionic N-hexadecyl-N,N-dimethyl-3-ammonium-1propanesulfonate (HPS) and the cationic cethyltrimethyl ammonium chloride (CTAC) surfactants was carried out [11] showing that, at stoichiometric surfactant concentrations, small local changes in the protein structure could be detected as monitored by tryptophan fluorescence. A complementary modeling work revealed that surfactant molecules bind to the BSA at a molar ratio of 5:1 [12]. This stoichiometry had been also earlier verified by means of calorimetry [26] and crystallographic studies [27]. 
Further, a systematic study focusing on the interaction of SDS with BSA at pH 5.4, near its isolectric point, was performed by SAXS and surface tension measurements [16] showing that below the critical aggregation concentration (cac) of $2.2 \mathrm{mM}$, the protein conformation remains unaltered. However, at $5 \mathrm{mM}$ SDS both the protein radius of gyration and maximum dimension increase, reflecting the beginning of BSA unfolding process. Moreover, surfactant concentrations higher than $10 \mathrm{mM}$ lead to the formation of micelle-like aggregates, and the modeling of SAXS curves is consistent with the "pearl necklace" model. The micelle-like aggregates are randomly distributed along the chain and grow in size, changing from sphere-like shape at $10 \mathrm{mM}$ SDS to spheroid-like form ( $v=1.3$, the longest to the shortest axes ratio) at $50 \mathrm{mM}$ SDS. Above $50 \mathrm{mM}$, the micelles stop growing, remaining in a prolate ellipsoidal shape. Another interesting result is associated to the fact that the size of the SDS micelles in the complex is always smaller than that observed for free-SDS micelles in solution at same surfactant concentration [13-15]. Our SAXS studies of interaction of SDS, HPS and CTAC with $\mathrm{BSA}$ at $\mathrm{pH} 7.0$ [18] have shown that the protein radius of gyration for $5 \mathrm{mM}$ SDS or HPS does not change significantly as compared to the pure protein while for $5 \mathrm{mM}$ CTAC an increase of radius of gyration takes place. Furthermore, the formation of micelles at higher CTAC concentrations was not as clear as for SDS or HPS.

The aim of our current work is to get more insights, by making use of electron paramagnetic resonance (EPR) techniques, on the changes produced in the BSA structure upon interaction with cationic CTAC. EPR spectroscopy with nitroxides reports on surfactants assembling in the presence of the protein allowing a comparison of the dynamic properties of pure and protein-associated micelle-like aggregates.

\section{MATERIALS AND METHODS}

\section{A. EPR experiments}

A Varian E-9 spectrometer operating at X-band $(9.4 \mathrm{GHz})$ was utilized in our investigations. The spectral parameters were as follows: microwave power, $20 \mathrm{~mW}$; modulation frequency, $100 \mathrm{KHz}$; modulation amplitude, 0.5-1.6 G; magnetic field scan, $100-160 \mathrm{G}$. Room temperature of $24^{\circ} \mathrm{C}$ was used.

Spin-labeled derivatives of stearic acids, 5- and 16-doxyl stearic acids (5-DSA and 16-DSA, respectively), were purchased from Aldrich Chem. Co. (Milwaukee, WI, U.S.A.). These spin labels have a nitroxide radical ring attached at C-5 and C-16 positions of the acyl chain, respectively. A small aliquot of stock spin label dissolved in ethanol $\left(5 \mathrm{mg} \cdot \mathrm{ml}^{-1}\right)$ was placed in an eppendorf tube and the solvent was evaporated under a stream of nitrogen gas. Samples containing 0.15 $\mathrm{mM}$ of BSA in phosphate buffer $20 \mathrm{mM} \mathrm{pH} 7.0$ and the appropriate amount of surfactant were added directly to the spin label thin film. The surfactants were introduced by dilution of a stock solution into the protein solution. The concentration of spin labels was estimated to be $0.1 \mathrm{mM}$ and the final concentration of BSA was of $0.15 \mathrm{mM}$. After labeling under gentle stirring, the samples were introduced into a capillary tube for EPR measurements.

\section{B. EPR analysis}

Rotational correlation times $\tau_{2 C}$ and $\tau_{2 B}$ were estimated from the heights of the spectral lines corresponding to nitrogen hyperfine interaction $\mathrm{M}_{I}=+1,0,-1$, and the linewidth of the central line $\Delta \mathrm{H}_{0}$, as described in references [28,29]. This estimate was performed for the nitroxides in pure micelles and in some few cases in the presence of protein where a single component was present in the experimental spectrum. The validity of this procedure is restricted to the fast motional regime of the nitroxide for rotational correlation times smaller than around $1 \mathrm{~ns}$. For strongly immobilized radicals observed in pure protein, spectral simulation was performed to obtain the values of $\mathrm{R}_{b a r}^{-1}$ associated to the diffusion tensor as described in [18]. Simulation of spectra was also performed for a few cases of pure micelles of CTAC.

EPR spectra simulations were performed by nonlinear least-squares (NLLS) fits, using the general slow-motional program [30,31]. The magnetic $\mathrm{g}$ and A tensors, are defined in a molecule-fixed frame, where the rotational diffusion rates around the $\mathrm{x}, \mathrm{y}$ and $\mathrm{z}$-axis are included [31]. By convention, the $\mathrm{x}$-axis points along the $\mathrm{N}-\mathrm{O}$ bond, the $\mathrm{z}$-axis is parallel to the $2 \mathrm{pz}$ axis of the nitrogen atom, and the $\mathrm{y}$-axis is perpendicular to $x$ and $z[28,30]$. The fitting program NLLS permits to estimate the rotational motion around the axes $\mathrm{x}, \mathrm{y}$, and $\mathrm{z}$ (the diffusion tensor R) for a single or multi-component EPR spectra. However, in order to reduce the number of free parameters and to simplify the simulation, we assume in some cases, as described in reference [18], an axially symmetric rotational diffusion tensor with $\mathrm{Rbar}=\left(\mathrm{R}_{\perp}^{2} \cdot \mathrm{R}_{/ /}\right)^{1 / 3}$ and a rotational anisotropy with $\mathrm{R}_{/ /}=10 \mathrm{R}_{\perp}$ [31]. The magnetic parameters $\mathrm{g}$ - and A-tensors, as well as the additional linewidths were optimized for pure BSA and pure micelles of CTAC.

\section{RESULTS AND DISCUSSION}

\section{A. Spin labeled micelles of pure surfactant}

In Fig. 1 the experimental EPR spectra at $24^{\circ} \mathrm{C}$ of stearic acid derivative spin label 5-DSA in phosphate buffer $20 \mathrm{mM}$ at $\mathrm{pH} 7.0$ in the presence of $10-40 \mathrm{mM}$ of cationic surfactant CTAC are shown. EPR spectra of both probes (similar spectra for 16-DSA were obtained; data not shown) in pure surfactant micelles correspond to a single component characterized by a correlation time which was estimated from the heights of the peaks correponding to $\mathrm{M}_{I}=+1,0,-1$ and the peak-to-peak linewidth of the central line $\mathrm{M}_{I}=0$. The values of $\tau_{2 C}$ were in the range 2.3-2.7 ns for 5-DSA and 0.50-0.56 ns for 16-DSA, respectively, increasing slightly at the higher CTAC concentration. $\tau_{2 B}$ values were in the range 1.3-1.6 ns for 5-DSA and $0.47-0.52 \mathrm{~ns}$ for 16-DSA. The larger anisotropy observed for 5-DSA (from the difference in $\tau_{2 B}$ and $\tau_{2 C}$ ) is probably associated to the larger motional restriction due to the location of 
the paramagnetic fragment of 5-DSA closer to the surfactant headgroup. The rotational motions of the spin labels in the presence of surfactant micelles were significantly lower than those in buffer solution as described in [18]. They are also lower for 5-DSA as compared to 16-DSA, in agreement with greater $\tau_{2 C}$ and $\tau_{2 B}$ for 5-DSA. In the range $10-40 \mathrm{mM}$ of surfactant the EPR spectra are essentially unchanged.

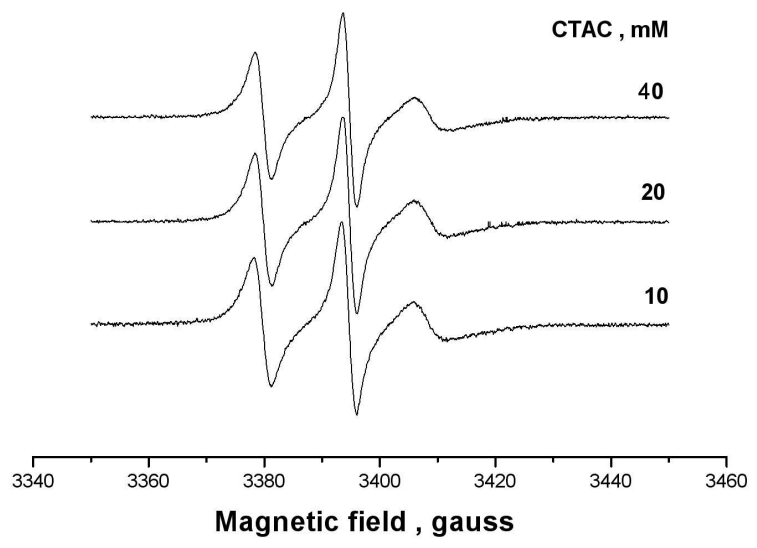

FIG. 1: EPR spectra of 5-DSA in solutions of pure CTAC at concentrations of 10,20 and $40 \mathrm{mM}$.

\section{B. Spin labeled BSA and BSA-CTAC system}

The binding of stearic-acid spin labels to BSA has been previously studied using the nonlinear least-squares simulation program [18,32]. The EPR spectral analysis made for stearic acid spin labels at different positions in the methylene chain showed an anisotropy in the rotational diffusion with a significantly faster motion around their long hydrocarbon chain, which was interpreted by the authors [32] assuming that the nitroxides are tightly held in some protein channels. In the present work, we focus on a comparison of the rotational diffusion of the spin labels in pure surfactant micelles, pure BSA solution and micelle-like aggregates of surfactant-BSA in order to obtain new data regarding the interaction of stearic acid and a cationic surfactant with the protein molecule. Experiments were performed in such a way that a fixed concentration of spin label, close to equimolar to the protein, was used. An assumption was made that the nitroxide stearate derivative would bind to the same protein high affinity sites as do both fatty acids or the used surfactants [5,12,18,26].

A limitation of this assumption is the fact that the association constants are quite different for anionic SDS, zwitterionic HPS and cationic CTAC, so that the competition of surfactant for the nitroxide binding site could be differentiated.

In Fig. 2 the experimental spectra of 5-DSA in BSA with different concentrations of CTAC in the range $2-40 \mathrm{mM}$ are shown. A general trend is observed and illustrated in this figure when the sample contained both protein and surfactant.
In this case two spectral components seem to be present, especially above some critical surfactant concentration which seems to be between 4 and $10 \mathrm{mM}$ of CTAC. Component 1 , the more immobilized one, represents the spin labels contacting BSA, and component 2 corresponds to the spin labels in micelle-like aggregates of BSA and surfactant. Fig. 3 presents the EPR spectra of 16-DSA bound to BSA in the absence and presence of 2-40 $\mathrm{mM}$ of CTAC. Here again it seems that two components are formed as a result of increasing the surfactant concentration. It is worthy of notice that neither for 5-DSA or 16-DSA the third spectral component associated to a fraction of spin labels freely tumbling in the buffer, and observed previously in the presence of SDS [18], was not present in the case of BSA-CTAC interaction. In the case of 16-DSA the formation of the second component associated to nitroxides partitioned in the protein-surfactant aggregates is more pronounced as compared to 5-DSA. In fact, at $7 \mathrm{mM}$ of surfactant a significant amount of component 2 is observed, while at $40 \mathrm{mM}$ of CTAC it seems that all of 16-DSA is shifted to a micellar environment (component 2) since no strongly immobilized signal (component 1) is observed (Fig. 3). In the case of cationic CTAC, which has a cmc around $1 \mathrm{mM}$ in our system, it is clear that low surfactant concentrations do not induce the appearance of free nitroxides signal in aqueous solution (Figs. 2 and 3) as occurs with SDS [18]. This is probably due to the fact that the association constant of CTAC to BSA is significantly smaller as compared to that for SDS and the cationic surfactant is not able to displace the nitroxides from the protein sites. Comparison of Figs. 2 and 3 shows that, apparently, increasing surfactant concentration leads to formation of micelle-bound nitroxide to a greater extent for 16-DSA as compared to 5-DSA. It is possible that this effect is associated to a higher binding constant of 5-DSA to the protein as compared to 16-DSA, or that due to the fact that the nitroxide moiety in 16-DSA is located at the end of the methylene chain it is easier to displace the nitroxide from the protein binding site. In fact, comparison of EPR spectra for the two nitroxides bound to pure protein (Figs. 2 and 3, pure protein) already shows a stronger immobilization in the case of 5-DSA (as will be shown later on from spectral simulation).

In Figs. 4 and 5 two experimental parameters are plotted as a function of CTAC concentration: $\Delta \mathrm{H}_{0}$, the linewidth of the central line in the EPR spectrum and $\mathrm{A}_{\max }$, the maximum splitting between the low field and high field lines. The linewidth for 5-DSA tends to be always higher as compared to that of 16-DSA (Fig.4) which is an evidence for slower motional rates. Besides that, for 5-DSA, in the presence of the protein, it seems that a biphasic change occurs as a function of surfactant concentration, an initial steep change in the range 2-4 mM of CTAC and a second one above $10 \mathrm{mM}$ surfactant. In the case of 16-DSA an unique change takes place around 10 $\mathrm{mM}$ of CTAC (Fig. 4). In the case of pure surfactants smooth variations of linewidth are observed for both nitroxides: above $10 \mathrm{mM}$ the linewidths tend to constant values. The maximum splitting shown in Fig. 5 also presents a dramatic change for 16-DSA as compared to 5-DSA above $10 \mathrm{mM}$ of CTAC. This is consistent with the transition of 16-DSA nitroxide to CTAC micelles above this concentration implying a very significant 


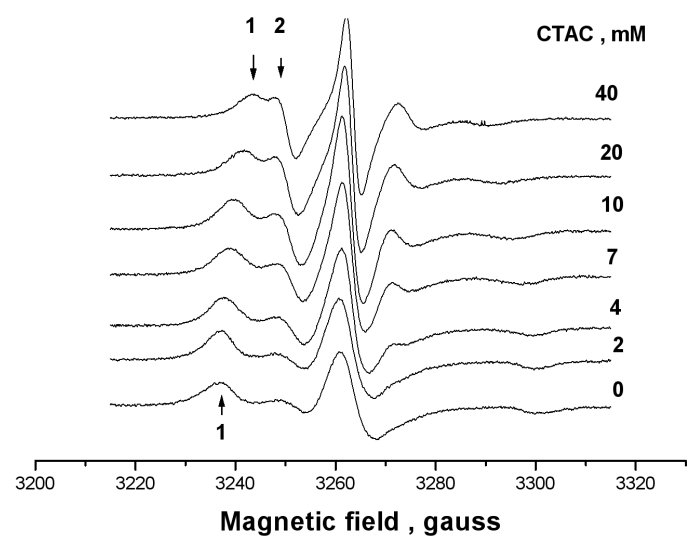

FIG. 2: EPR spectra of 5-DSA in solutions of BSA $0.15 \mathrm{mM}$ in phosphate buffer $20 \mathrm{mM} \mathrm{pH} 7$ at indicated concentrations of cationic surfactant CTAC. The arrows indicate the protein bound component 1 and micelle bound component 2 .

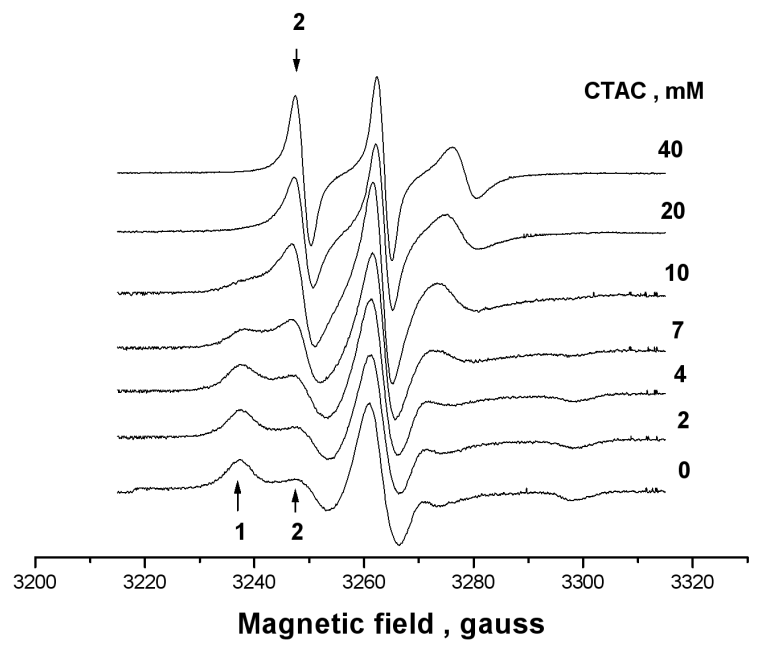

FIG. 3: EPR spectra of 16-DSA in solutions of BSA $0.15 \mathrm{mM}$ in phosphate buffer $20 \mathrm{mM} \mathrm{pH7}$ and indicated concentrations of cationic surfactant CTAC. The arrows indicate the protein bound component 1 and micelle bound component 2 .

increase in rotational motion for the paramagnetic fragment attached to the $16^{\text {th }}$ carbon of the nitroxide methylene chain. The estimated rotational correlation times from the intensities of the components are $\tau_{2 C}=1.7 \mathrm{~ns}$ and $\tau_{2 B}=1.3 \mathrm{~ns}$. At the same time motional restriction persists for 5-DSA even at 40 $\mathrm{mM}$ of CTAC.

\section{EPR spectral simulation using the NLLS program}

Spectral simulation was performed with the NLLS program for several samples described above. In the case of pure CTAC

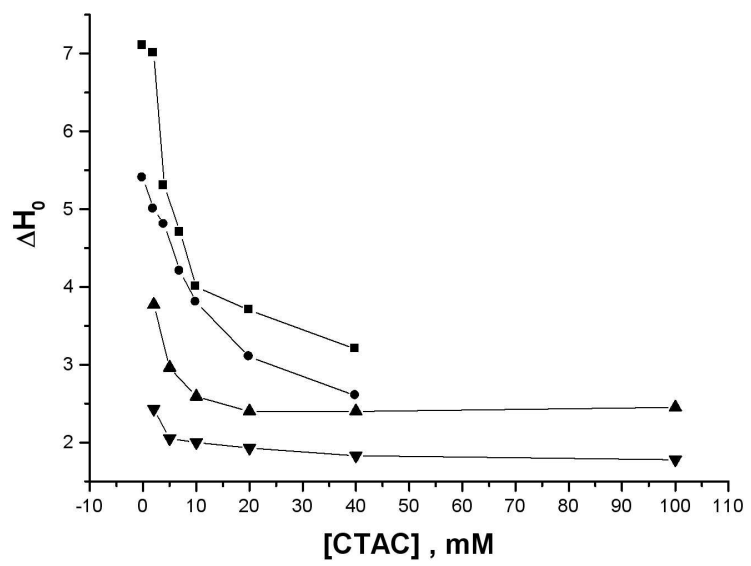

FIG. 4: Variation of linewidth of the central line $\mathrm{M}_{I}=0$ as a function of CTAC concentration for the BSA-CTAC and pure CTAC systems. Squares correspond to 5-DSA and circles to 16-DSA in BSA-CTAC system; triangles correspond to 5-DSA and up-triangles to 16-DSA in pure CTAC system. The lines were drawn just as a guide for the eye.

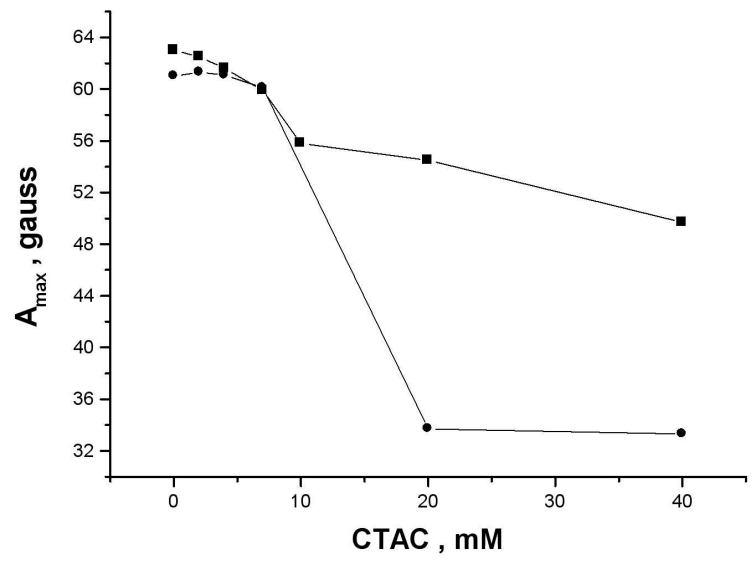

FIG. 5: Variation of the maximum hyperfine splitting in the EPR spectra of 5-DSA (squares) and 16-DSA (circles) as a function of CTAC concentration. Data correspond to spectra presented in Figs. 1 and 3 . The lines were drawn just a guide for the eye.

micelles, at $40 \mathrm{mM}$, the spectra for 5-DSA (Fig. 1) and 16DSA were simulated. Initially the magnetic tensors were optimized leading to best fit parameters as follows: principal components for the g-tensor, $\mathrm{g}_{x x}, \mathrm{~g}_{y y}$ and $\mathrm{g}_{z z}$, were equal to $2.0085,2.0062$ and 2.0030 for 5-DSA, and 2.0090, 2.0062 and 2.0028 for 16-DSA; principal components for the nitrogen hyperfine tensor, $\mathrm{a}_{x x}, \mathrm{a}_{y y}$ and $\mathrm{a}_{z z}$, were equal to $6.2,5.9$ and 33.3 gauss for 5-DSA and 6.8, 5.2 and 33.2 gauss for 16-DSA. The average nitrogen hyperfine tensor was $\mathrm{a}_{0}=15.13$ gauss for 5-DSA and 15.07 gauss for 16-DSA showing very similar envinronment in terms of polarity for both nitroxides. The magnetic parameters were fixed after initial optimization 
and a model of axial symmetry for motion $\left(\mathrm{n}_{x y}=0\right)$ with $\mathrm{n}=1$ was used. The value of $n$ was varied later on, and it was noticed that its variation in a broad range (down to $n=0$ ) did not changed significantly the quality of the fit $\left(\chi^{2}\right.$ value) while the rotational diffusion $\mathrm{R}_{b a r}^{-1}$ could be changed significantly. This means that in the fast motional regime, characteristic for nitroxides in pure micelles, the degree of anisotropy associated to $\mathrm{n}$ is largely undetermined. The slow component dominates the spectral features, mantaining constant $\mathrm{R}_{\perp}$. In other words, it is difficult to obtain a very precise value for it from the simulation. In the case of 5-DSA the fit was good enough with the gaussian and lorentzian linewidth parameters set to 0 and the rotational diffusion obtained was $\mathrm{R}_{b a r}^{-1}=6.2 \mathrm{~ns}$. The corresponding rotational correlation time $\tau=\mathrm{R}_{b a r}^{-1} / 6=1 \mathrm{~ns}$. In the case of 16-DSA the same model was used but the fit was improved using linewidth parameters as gib0 $=1.46$ and $\mathrm{lb}=0.1$. The rotational diffusion was $\mathrm{R}_{\text {bar }}^{-1}=1.2 \mathrm{~ns}$ with corresponding rotational correlation time of $0.2 \mathrm{~ns}$. It is interesting that for both 16-DSA and 5-DSA the rotational correlation times obtained from the simulation were a factor of 2 lower as compared to the values obtained from the intensity ratios as described above. However, as mentioned before, change of the $n$ value towards its value for the isotropic model $(n=0)$ leads to a reduction of $\mathrm{R}_{b a r}$ with consequent convergence of the two correlation times, from simulation and intensity ratios, to the same value.

Next in the simulation the strongly immobilized spectra for 5- and 16-DSA bound to pure BSA (absence of surfactant) were considered. For 5-DSA the magnetic parameters were $\mathrm{g}_{x x}, \mathrm{~g}_{y y}$ and $\mathrm{g}_{z z}$ equal, respectively, to 2.0086, 2.0063 and 2.0025 , and $\mathrm{a}_{x x}, \mathrm{a}_{y y}$ and $\mathrm{a}_{z z}$ equal to $6.9,5.4$ and 33.8 gauss. The average hyperfine tensor $\mathrm{a}_{0}=15.4$ gauss suggests that 5 DSA bound to the protein monitores an envinronment which is slightly more polar as compared to that observed in pure CTAC micelle. These values are identical to those obtained in reference [32]. Axial symmetry for motion was assumed with $\mathrm{n}=0.85$ and $\mathrm{n}_{x y}=0$ as in [32]. The gaussian linewidth parameter gib0 for the best fit was 4.7 while the lorentzian parameter was kept zero. The rotational diffusion parameter $\mathrm{R}_{b a r}^{-1}$ for the best fit was $98 \mathrm{~ns}$ leading to a rotational correlation time of $16.6 \mathrm{~ns}$. In the case of 16-DSA bound to pure BSA the spectrum was adequately simulated to identical g-tensor values and the only hyperfine component which was different in comparison to 5-DSA is $\mathrm{a}_{z z}$ which was 33.2. This gives an average value $\mathrm{a}_{0}=15.3$ very similar to that obtained for 5-DSA. Additional gaussian and lorentzian linewidths were optimized and the rotational diffusion parameter for the best fit was $36 \mathrm{~ns}$ leading to a rotational correlation time of $6 \mathrm{~ns}$. In Fig. 6 the pure protein spectra for both 5-DSA and 16-DSA are presented together with the experimental EPR spectra. Evidently the fits are quite good. It is also worth of mentioning the characteristic rotational correlation time that would be expected for intact native BSA based on SAXS data as well as from fluorescence anisotropy decay experiments $[18,33]$. Using the Stokes-Einstein relation for the rotational correlation time $\tau_{c}=\eta$.V/RT, where $\eta$ is the solution viscosity, $V$ the particle volume, $\mathrm{R}$ the universal gas constant, and $\mathrm{T}$ the temperature, and assuming that the volume of BSA is given as that of a sphere of radius equal to $38 \mathrm{~A}$, will give an estimate of $\tau_{c}$ of 51 ns [18]. From fluorescence anisotropy decay of tryptophans of BSA this value is $42 \mathrm{~ns}$ [33]. In this way, our values obtained in the present work for the rotational correlation times of the nitroxides bound to BSA are certainly an average value which include some contribution of the motion of the paramagnetic fragment relative to the whole protein particle. This explains the lower value of $\tau_{c}$ obtained from EPR spectral simulation as compared to the expected value for the protein.

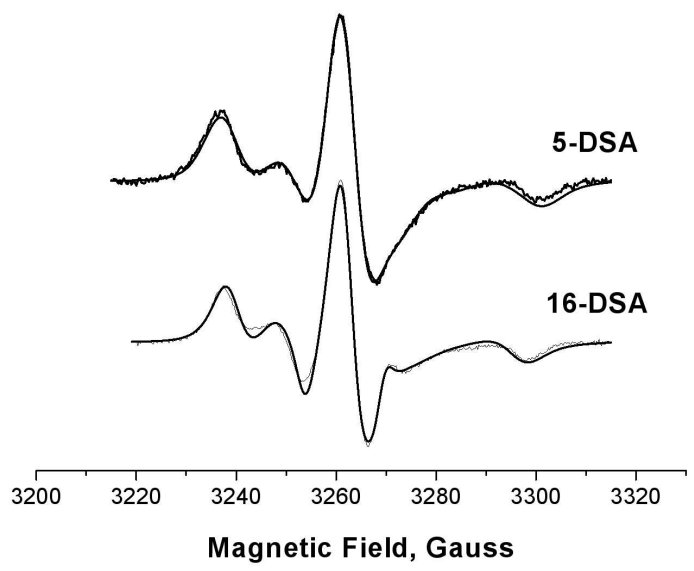

FIG. 6: Experimental and simulated EPR spectra for 5-DSA and 16DSA nitroxides bound to pure BSA samples. Protein concentration $0.15 \mathrm{mM}$.

Finally, the spectrum for 16-DSA in the mixed system BSA plus CTAC $40 \mathrm{mM}$ was simulated. The best fit model was similar to that in pure CTAC micelles giving $\mathrm{g}_{x x}, \mathrm{~g}_{y y}$ and $\mathrm{g}_{z z}$ of 2.0082, 2.0061 and 2.0030, and $\mathrm{a}_{x x}, \mathrm{a}_{y y}$ and $\mathrm{a}_{z z}$ equal to $6.9,5.4$ and 32.3 gauss. The average hyperfine tensor was $\mathrm{a}_{0}$ $=14.86$ gauss, again indistinguishable from the value in pure CTAC micelles. The rotational diffusion $\mathrm{R}_{b a r}^{-1}$ was equal to 3.7 ns, a value higher as compared to that in pure CTAC micelles, implying that in the presence of protein the motion of 16-DSA in the micelles becomes more restricted. Work to simulate the remaining EPR spectra of the mixture BSA-CTAC is in progress to evaluate the contributions of the two populations of nitroxides, one bound to the protein and the second located in the protein-bound micelles.

It is worthy of mentioning the work described in [13] where the authors used a fluorescence probe to monitor changes in BSA upon addition of SDS and CTAB as well as a neutral surfactant. This work is quite similar to our EPR experiments described above. The authors used pyrene as a fluorescent probe and monitor the $I_{1} / I_{3}$ ratio of vibrational bands to access probe location and changes upon surfactant addition. In the absence of surfactant the probe binds to BSA yielding a value of $\mathrm{I}_{1} / \mathrm{I}_{3}$ of 1.3 , lower than the value in water (1.8) and slightly higher than that in pure SDS micelles (1.2). This indicates binding of pyrene to hydrophobic sites in BSA. Measurements of pyrene fluorescence lifetimes showed that in the presence of BSA for both SDS and CTAB (and the neutral surfactant used in this 
study as well) a significant increase of lifetime was observed and, as the concentration of surfactant is increased, the lifetime tends to the value observed in pure micelles, which is higher as compared to aqueous solution due to the protection from water of the chromophore and possibly protection from oxygen dissolved in water. The authors found that the lifetime starts to decrease at approximately $1 \mathrm{mM}$ of surfactant regardless of surfactant type or buffer $\mathrm{pH}$. This concentration is associated to massive cooperative surfactant binding that for SDS is around 1-2 mM. Aggregation numbers of surfactants in the presence of BSA were also estimated through static and timeresolved fluorescence quenching. These numbers are much smaller (approximately half) as compared to pure surfactants and seem to be independent of buffer composition. In comparison with SDS, the aggregation numbers of CTAB are smaller, whereas for free micelles the CTAB micelles are larger than the SDS ones. This means that the CTAB micelles bound to BSA are smaller as compared to SDS ones.

\section{CONCLUSIONS}

For pure micelles a single component is observed in the EPR spectra characteristic of the mobility of nitroxides in micelles, where 16-DSA has rotational correlation times in the subnanosecond region of fast reorientational motion while 5DSA has reorientational correlation times of a few nanosecond in the limit of validity of the fast motion regime. Simulated values for $\tau_{c}$ are a factor of 2 lower as compared to values estimated from component intensity ratios. The EPR data obtained with spin labels strongly coupled to the polypeptide chains indicated mobility increases even when the detergent concentrations are as low as $2 \mathrm{mM}$. In the presence of the protein the EPR spectra correspond to two different spin label populations, with different mobilities and associated to one population in close contact to the protein and probably accomodated in the high affinity protein binding sites and a second one distributed in the micellar aggregates and sensitive to the presence of protein as revealed by EPR spectral parameters.

The EPR data indicated also more mobility for pure surfactant micelles than for "micelle-like" aggregates in presence of protein. For CTAC and 5-DSA even at the highest surfactant concentration of $40 \mathrm{mM}$ an immobilized component was still present in the spectrum, while for 16-DSA, the nitroxide was, apparently, completely transferred to a micellar envinronment. This is probably consistent with a lower affinity of this nitroxide to the protein as observed from its higher mobility observed from a lower rotational correlation time in the absence of surfactant for the pure protein. For those spin labels inside the "micelle-like" aggregates a decrease of motion compared to the motion in pure micelles would be expected, since the spin labels are subject to stronger interactions. The interaction between "micelle-like" aggregates and the protein also reduces the overall rotational motion of these micelles.

\section{Acknowledgments}

The authors thank to CNPq and FAPESP Brazilian agencies for partial financial support.
[1] D. Carter, B. Chang, J. X. Ho, K. Keeling, and Z. Krishnasami, Eur. J. Biochem. 226, 1049 (1994).

[2] J. R. Brown, P. Shockley. Lipid-Protein Interactions, vol. 1, Wiley, New York, 1982.

[3] T. Peters, All about albumins: biochemistry, genetics and medical applications. San Diego, Academic Press, 1996.

[4] X. Min He, D. Carter, Nature 358, 209 (1992).

[5] S. Curry, H. Mandelkow, P. Brick, and N. Franks. Nat. Struct. Biol. 5, 827 (1998).

[6] C. Tanford, The hydrophobic effect: formation of micelles and biological membranes, Wiley, New York, 1973.

[7] N. J. Turro, K. P. Ananthapadmanabhan, X. G. Lei, and M. Aronson, Langmuir 11, 2525 (1995).

[8] M. N. Jones, D. Chapman, Micelles, monolayers and biomembranes, Wiley-Riss, New York, 1995.

[9] J .A. Reynolds, C. Tanford, J. Biol. Chem. 245, 5161 (1970).

[10] H. Durchschlag, K. J. Tiefenbach, S. Gebauer, and R. Jaenicke, J. Mol. Struct. 563, 449 (2001).

[11] E. L. Gelamo, M. Tabak, Spectrochem. Acta, 56, 2255 (2000).

[12] E. L. Gelamo, C.H.T.P. Silva, H. Imasato, and M. Tabak, Biochim. Biophys. Acta, 1594, 84 (2002).

[13] M. Vasilescu, D. Angelescu, M. Almgren, and A. Valstar, Langmuir, 15, 2635 (1999).

[14] S. H. Chen, J. Teixeira, Phys. Rev. Lett. 57, 2583 (1986).
[15] X. H. Guo, N. M. Zhao, S. H. Chen, and J. Teixeira, Biopolym. 29, 335 (1990).

[16] S. F. Santos, D. Zanette, H. Fischer, and R. Itri, J. Colloid and Interface Sci. 262, 400 (2002).

[17] S. Shinagawa, M. Sato, K. Kameyama, and T. Takagi, Langmuir 10, 1690 (1994).

[18] E. L. Gelamo, R. Itri, A. Alonso, J. V. da Silva, and M. Tabak, J. Coll. Int. Sci.277, 471 (2004).

[19] R. E. Tanner, B. Herpigny, S. H. Chen, and C. K. Rha, J. Chem. Phys. 76, 3866 (1982).

[20] A. Valstar, M. Almgren, W. Brown, and M. Vasilescu, Langmuir, 16, 922 (2000).

[21] A. K. Morén, O. Regev, and A. Khan, J. Coll. Int. Sci. 222170 (2000).

[22] A. K. Morén, A. Khan, J. Coll. Int. Sci. 218, 397 (1999).

[23] S. Ghosh, A. Banerjee, Biomacromol. 3, 9 (2002).

[24] M. D. Lad, V. M. Ledger, B. Briggs, R. J. Green, and R. A. Frazier, Langmuir 19, 5098 (2003).

[25] D. Kelley, D. J. McClements, Food Hydrocoll. 17, 73 (2003).

[26] A. D. Nielsen, K. Borch, and P. Westh, Biochim. Biophys. Acta 1479, 321 (2000).

[27] S. Curry, B. Peter, N. P. Franks, Biochim. Biophys. Acta 1441, 131 (1991).

[28] L. J. Berliner, editor, Spin labeling. Theory and applications, 
Academic Press, New York, 1976.

[29] V. E. Yushmanov, M. Tabak, J. Coll. Int. Sci.191, 384 (1997).

[30] D. J. Schneider, J. H. Freed, Biological Magnetic Resonance, v.8, L. J. Berliner and J. Reuben eds, Plenum Press, New York. 1-76, 1989.

[31] D. E. Budil, S. Lee, S. Saxena, and J. H. Freed, J. Magn. Reson. A 120, 155 (1996).
[32] M. T. Ge, S. B. Rananavare, and J. H. Freed, Biochim. Biophys. Acta, 1036, 228 (1990).

[33] J. R. Lakowicz, Principles of fluorescence spectroscopy, $2^{\text {nd }}$ edition, Kluwer Academic/Plenum Publishers, New York, 1999. 